\title{
Cavity-enhanced noncollinear high-harmonic generation
}

\author{
Maximilian Högner, ${ }^{1,2}$ Tobias Saule,${ }^{1,2}$, Stephan HeinRICH ${ }^{1,2}$ \\ Nikolai Lilienfein, ${ }^{1,2}$ Dominik EsSer, ${ }^{3}$ Michael Trubetskov, ${ }^{1}$ \\ Volodymyr PERVAK, ${ }^{2}$ AND IOACHIM PUPEZA $1,2,{ }^{*}$ \\ ${ }^{1}$ Max-Planck-Institut für Quantenoptik, Hans-Kopfermann-Straße 1, 85748 Garching, Germany \\ ${ }^{2}$ Ludwig-Maximilians-Universität München, Am Coulombwall 1, 85748 Garching, Germany \\ ${ }^{3}$ Fraunhofer-Institut für Lasertechnik, Steinbachstraße 15, 52074 Aachen, Germany \\ *ioachim.pupeza@mpq.mpg.de
}

\begin{abstract}
Femtosecond enhancement cavities have enabled multi-10-MHz-repetition-rate coherent extreme ultraviolet (XUV) sources with photon energies exceeding $100 \mathrm{eV}$ - albeit with rather severe limitations of the net conversion efficiency and of the duration of the XUV emission. Here, we explore the possibility of circumventing both these limitations by harnessing spatiotemporal couplings in the driving field, similar to the "attosecond lighthouse," in theory and experiment. Our results predict dramatically improved output coupling efficiencies and efficient generation of isolated XUV attosecond pulses.
\end{abstract}

(C) 2019 Optical Society of America under the terms of the OSA Open Access Publishing Agreement

\section{Introduction}

In recent years, femtosecond enhancement cavities (ECs) have matured to an enabling technology for precision metrology with coherent radiation in the vacuum and extreme ultraviolet (VUV, XUV) spectral regions. ECs are passive optical resonators that can be efficiently excited over a broad optical band, usually in the near-infrared (NIR), by the pulse train of a (post-amplified, phase-stabilized) modelocked laser. This results in a circulating pulse with an energy enhanced by a few orders of magnitude with respect to that of the seeding pulses, affording intensities high enough to drive high-order harmonic generation (HHG) in gases at repetition rates of several tens of $\mathrm{MHz}[1,2]$.

Direct evidence of the temporal coherence of the emerging harmonic spectrum $[3,4]$ has demonstrated the viability of transferring NIR frequency combs to the VUV/XUV with ECs, thus paving the way towards precision frequency metrology of electronic transitions [5]. Very recently, geometrically coupling out the harmonic radiation through an on-axis opening in the mirror following the HHG focus [6-8] enabled MHz-HHG with photon energies high enough to liberate core electrons from metals via single-photon photoelectron spectroscopy (PES). This led to the first space-charge-free PES experiments at multi-MHz repetition rates [8,9], in particular also with attosecond temporal resolution [8]. Significant efforts have addressed the understanding of cavity-enhanced HHG conversion efficiency limitations related to plasma nonlinearity [10-12] and plasma cumulative effects $[13,14]$. Accelerating the gas to provide (nearly) single-pass conditions even at several tens of $\mathrm{MHz}$ has been shown to strongly mitigate the latter limitation, resulting in $\mathrm{mW}$-level VUV frequency combs [13]. In addition, novel, nonlinearity-optimized ultrashort-pulse enhancement regimes $[12,15]$ promise a path to circumvent the blueshift-related intensity clamping [10-12]. Altogether, these recent advances indicate a vast potential of improving the intracavity conversion efficiency.

Yet, despite of the growing attention dedicated to this laser architecture uniquely combining high XUV photon energies with high repetition rates, two limitations remain without solution to this day. Firstly, geometric output coupling (OC) employing the fundamental transverse mode of the EC $[6-8,14]$ - the most broadband OC technique demonstrated so far - suffers from poor 
efficiency [16]. Noncollinear methods or methods employing spatially tailored resonator modes have been recognized early on as promising alternatives [17-21]. Secondly, the direct generation of isolated attosecond pulses (IAP), as desirable for endowing time-resolved PES with attosecond resolution over durations of hundreds of femtoseconds [22-24], has remained out of reach due to the limited spectral coverage of today's ECs [25]. In this letter, we demonstrate a new means of control over the transverse mode of a high-finesse, broadband femtosecond EC, offering a route towards circumventing both above-mentioned limitations.

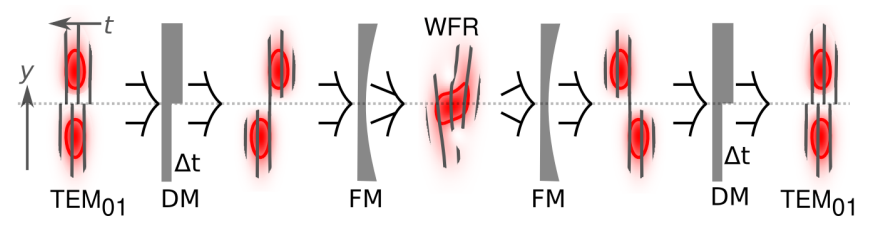

Fig. 1. Working principle of transverse mode gating: one lobe of a $\mathrm{TEM}_{01}$ resonator mode (wave fronts indicated by gray lines) is delayed by an odd number of half cycles using a half-sided delay mirror (DM). After a focusing mirror (FM), this leads to an on-axis maximum around the focus where the high-harmonic generation gas target is placed, with a wavefront rotation (WFR) adjustable by the step height of the DM. After the focus, the alteration of the mode is reversed, permitting low-diffraction-loss propagation in the resonator. Operating with negligible WFR affords high-efficiency geometric output coupling of the harmonic radiation emitted by all NIR half cycles. A larger WFR can be used to spatially isolate the harmonic emission of a single NIR half cycle, thus gating an isolated attosecond pulse.

\section{Methods}

To this end, we employ the TMG (transverse mode gating) method introduced in [26]: A TEM ${ }_{01}$ resonator mode is excited, which consists of two lobes of opposite phase, spatially separated by an intensity minimum (see Fig. 1). One of the lobes is delayed with respect to the other by means of an EC mirror with a stepped surface profile [27], introducing spatiotemporal coupling. Similar to noncollinear optical gating $[19,28,29]$ and the attosecond lighthouse [30-33], this leads to wave-front rotation (WFR), involving contributions from spatial chirp and pulse front tilt, in a subsequent focal region. Moreover, a delay equal to an odd number of optical half cycles results in a single, on-axis maximum around the focus [17]. The delay is reversed by a second, identical mirror located such that the first mirror is imaged onto it in good approximation $[26,27]$. This ascertains that the recycled field can overlap constructively with the original $\mathrm{TEM}_{01}$ mode, allowing the field to circulate with negligible losses inside a resonator housing this configuration. A mirror with a small on-axis opening can be used for efficient $\mathrm{OC}$ of the radiation generated in a gas target placed in the focal region, while introducing negligible losses to the circulating pulse thanks to the on-axis intensity minimum of the $\mathrm{TEM}_{01}$ mode $[17,21]$.

This technique is advantageous for cavity-enhanced HHG even when temporal gating is not required: By choosing a delay of just one half cycle and using multi-cycle circulating pulses, OC efficiencies $>40 \%$ can be expected irrespective of the position of the gas target, resolving a major limitation on the overall conversion efficiency of EC-based XUV sources [16]. In contrast to similar approaches based on quasi-imaging [20,21], this technique does not require operation in the middle of the stability range of the resonator, so that limitations on the peak power of the circulating pulses are relaxed by allowing for larger spot sizes on the mirrors (see Appendix I).

When choosing higher values of the delay and using few-cycle circulating pulses, the WFR angularly disperses the individual bursts of the generated XUV radiation, allowing the selection of an IAP by spatial filtering. The delay between the two lobes is intrinsically stable due to the 
monolithic step mirrors, as opposed to other proposed schemes for noncollinear HHG in ECs employing two separate cavities or crossing two pulses circulating in a single EC of twice the length [17-19].

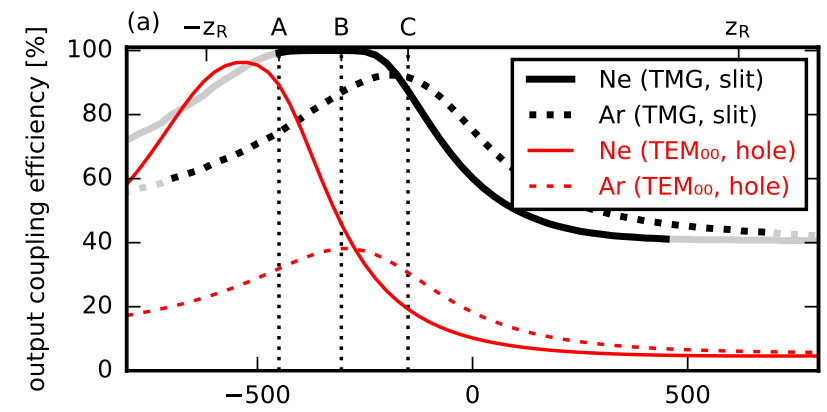

(b)

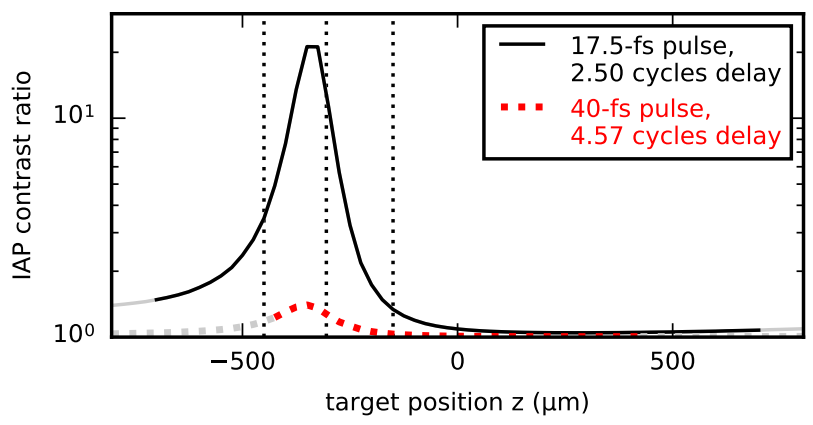

Fig. 2. Figures of merit for transverse mode gating. (a) Output coupling efficiency with the TMG mode (bold black lines) vs. hole output coupling with the fundamental mode (thin red lines), for HHG in argon (H33 at a peak intensity of $1.5 \times 10^{14} \mathrm{~W} / \mathrm{cm}^{2}$, dashed lines) and neon (H79 at a peak intensity of $3.0 \times 10^{14} \mathrm{~W} / \mathrm{cm}^{2}$, solid lines), with 40 -fs pulses centered at $1025 \mathrm{~nm}$ and the output coupling slit/hole dimensions chosen so that the round-trip loss in both cases remains below 1\%. (b) Expected on-axis inter-burst contrast ratio versus gas target position using 17.5-fs pulses and an accordingly chosen delay (solid black line) and with the demonstrated 40-fs pulses (dashed red line), in both cases computed for $\mathrm{H} 79$ in neon at a peak intensity of $3.0 \times 10^{14} \mathrm{~W} / \mathrm{cm}^{2}$. The gray continuations of the curves identify when pulse energies $>80 \mu \mathrm{J}$ are needed to reach the corresponding peak intensities. The vertical dotted lines labeled with A, B and C mark the $z$ position for the data shown in Figs. 5(b), 4 and 5(d), respectively.

\section{Results}

The spatial divergence of the resulting XUV beamlets is determined by the position of the gas target relative to the focus. The divergence must be small to ensure efficient OC at the opening in the mirror and, in the case of IAP production, to avoid spatial overlap between the individual beamlets. Therefore, the gas target position is a critical parameter for both modes of operation. The smallest divergence is achieved when the wave-front curvature of the driving beam compensates for the wave-front curvature induced by the transverse intensity gradient via the intensity-dependent phase of the dipole response [16,34]. In the following, we theoretically investigate the effect of the target position on the OC efficiency and the gating efficiency.

For the first application, i.e., applying the scheme with negligible WFR to improve the OC efficiency of the harmonic radiation, we compute the $\mathrm{OC}$ efficiency for different positions 
of the gas target, assuming a TMG mode with 40-fs Gaussian pulses centered at $1025 \mathrm{~nm}$, a step height of $0.26 \mu \mathrm{m}$ corresponding to a delay of 0.5 cycles, focused to a spot size of $w_{0, x} \times w_{0, y}=17.2 \times 11.8 \mu \mathrm{m}^{2}$, and an OC mirror with a 7.31-mrad-angular-width slit (e.g., a 0.731-mm-broad slit in a OC mirror located $100 \mathrm{~mm}$ behind the focus), chosen for a maximum round-trip loss of 1\%. The numerical model is described in detail in Appendix A. We compare the method to OC using a symmetric fundamental Gaussian mode focused down to the same focal spot area, using an OC mirror with a hole, with an angular diameter chosen for the same round-trip loss $(2.30 \mathrm{mrad})$. Because the divergence of the XUV beamlets depends on the harmonic order, intensity and target gas [16], we consider two cases: OC of the $33^{\text {th }}$ harmonic produced in argon $\left(39.9 \mathrm{eV}\right.$, compare [8]), with a peak intensity of $1.5 \times 10^{14} \mathrm{~W} / \mathrm{cm}^{2}$ in the target plane, and of the $79^{\text {th }}$ harmonic produced in neon $(95.6 \mathrm{eV}$, compare [26]), with a peak intensity of $3.0 \times 10^{14} \mathrm{~W} / \mathrm{cm}^{2}$. The results are plotted in Fig. 2(a). Using the fundamental mode results in OC efficiencies below 18\% (argon) and 10\% (neon) for gas targets placed near the focus. Better OC efficiency can be achieved far in front of the focus, at the cost of XUV generation efficiency [16]. Using the TMG mode allows for OC efficiencies $>40 \%$ irrespective of the target position, thereby considerably alleviating this trade-off.

To model the temporal gating performance when operating with WFR, we numerically approximated the inter-burst intensity contrast ratio of the attosecond pulse trains emitted on-axis versus gas target position (see Appendix C), assuming the same central wavelength and focal spot size as before and a peak intensity of $3.0 \times 10^{14} \mathrm{~W} / \mathrm{cm}^{2}$ in a neon gas target. For the simulations, we used the parameters from our experiment (40-fs pulses and a delay of 4.57 cycles), as well as parameters which seem technologically within reach (17.5-fs pulses [25], using a delay of 2.5 cycles). As can be seen in Fig. 2(b), generation of isolated attosecond pulses around the $79^{\text {th }}$ harmonic order should be possible. For this, the target must be placed $350 \mu \mathrm{m}$ in front of the focus.

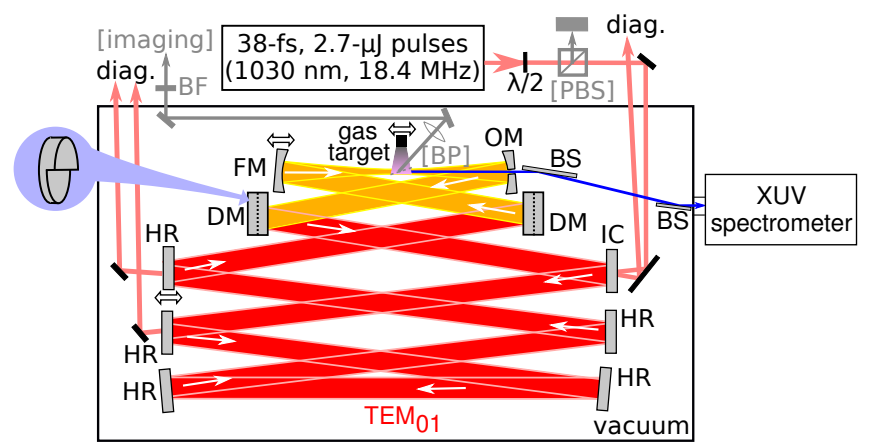

Fig. 3. Experimental setup. IC: input coupler, HR: highly reflective mirror, DM: HR delay mirror with a stepped surface profile, FM: focusing HR mirror, OM: FM with an on-axis hole for output coupling, BS: IR/XUV beam splitter, diag.: diagnostics. Optional, for imaging the spatial dispersion: BP: Brewster plate, BF: optical bandpass filter, and an attenuator consisting of a half-wave plate $(\lambda / 2)$ and a polarizing beam splitter (PBS)

To demonstrate the feasibility of the method, we set up a 10-mirror, 16.3-m-long EC (see Fig. 3) comprising two spherical focusing mirrors (radius of curvature $R=300 \mathrm{~mm}$ ), two delay mirrors with a stepped [27] surface profile $(2.34 \mu \mathrm{m}$ height, corresponding to a delay of 2.57 cycles at a wavelength of $1025 \mathrm{~nm}$ ), an input coupler with a reflectivity of $97.2 \%$ and five highly reflective plane mirrors. The EC is seeded by a laser system providing a $18.4-\mathrm{MHz}$, zero-offset-frequency train of $2.7-\mu \mathrm{J}, 38$-fs pulses centered at $1030 \mathrm{~nm}$, which is described in detail in [8]. The highly reflective mirror coatings were designed for a bandwidth supporting $\sim 30$-fs pulses. We operated the EC near the inner stability edge to achieve large mode areas 
and thereby small peak intensities on the mirrors when operating at high peak powers [35]. By selecting a proper combination of mirror coatings, we obtained a cavity with a preferred offset frequency of zero [36], which was verified by measuring the average power in the empty cavity for different values of the seeding comb offset frequency.

The radii of the focal spot were $w_{0, x} \times w_{0, y}=17.2 \times 11.8 \mu \mathrm{m}^{2}$, the same value as used for the simulations shown in Fig. 2. We measured $80-\mu \mathrm{J}$ pulses with a spectrum centered at $1025 \mathrm{~nm}$ in the empty cavity, at a finesse of 188 (round-trip loss of $0.5 \%$ ). While output coupling with a quasi-imaging method [20,21] would allow for peak powers up to $1.17 \mathrm{GW}$ (assuming an EC of the same length and mirrors with the same damage threshold), our setup is, owing to the operation near the inner stability edge, scalable beyond this value, reaching $1.88 \mathrm{GW}$ with a peak intensity of $7.4 \times 10^{9} \mathrm{~W} / \mathrm{cm}^{2}$ on the mirrors (see Appendix I).

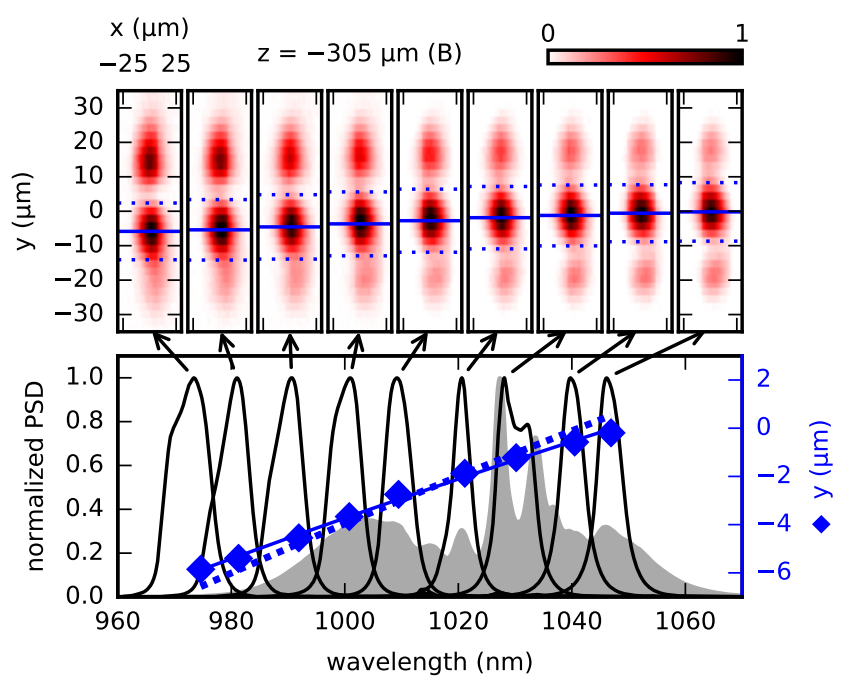

Fig. 4. Top: Transverse intensity profiles of the cavity mode $305 \mu \mathrm{m}$ in front of the focus, imaged with the arrangement shown in Fig. 3, and filtered for different wavelengths. The blue lines mark the position (solid) and $1 / e^{2}$-width (dotted) of Gaussian functions fitted to the central lobe of the horizontally integrated profile. Bottom: The intracavity spectrum (gray) and, for each depicted profile, the corresponding spectrum transmitted through the bandpass filter (black, normalized). The diamonds mark the central lobe positions and corresponding central wavelengths of the filtered spectra. A linear fit (blue solid line) of the lobe position vs. frequency yields a spatial dispersion of $-44 \mu \mathrm{m} / \mathrm{PHz}$, compared to a theoretical value of $-53 \mu \mathrm{m} / \mathrm{PHz}$ (blue dotted line).

To experimentally confirm the formation of spatial chirp around the focus, we attenuated the seed power and imaged a plane slightly in front of the focus $(305 \mu \mathrm{m})$ via a 100 -nm-thick silicon nitride plate inserted under close to Brewster's angle in the cavity beam (Fig. 3, gray). We employed a tilted narrow-band transmissive optical bandpass filter placed before a beam profiling camera to record the imaged profiles for different spectral portions of the transverse mode, confirming spatial dispersion (Fig. 4). We obtain a value of $\gamma=\mathrm{d} y / \mathrm{d} \omega=-44 \mu \mathrm{m} / \mathrm{PHz}$ (blue solid line in Fig. 4, lower panel), in agreement with the prediction of the numerical model of $-53 \mu \mathrm{m} / \mathrm{PHz}$ (blue dotted line in Fig. 4, lower panel, see Appendix D).

From the measured spatial dispersion $\gamma$, the lobe radius $w_{y}=(8.8 \pm 0.4) \mu \mathrm{m}$ and spectral width $(\Delta \omega=58.9 \mathrm{THz}$ for a Fourier-limited 40-fs Gaussian pulse), we can approximate the frequency gradient $\mathrm{d} \omega / \mathrm{d} y=\gamma /\left(\gamma^{2}+\left(w_{y} / \Delta \omega\right)^{2}\right)=-1.81 \mathrm{THz} / \mu \mathrm{m}$ [37] and the spatial chirp $\mathrm{d} l / \mathrm{d} y=-2 \pi c \omega_{c}^{-2} \mathrm{~d} \omega / \mathrm{d} y=1.15 \mathrm{~nm} / \mu \mathrm{m}$ for a central frequency $\omega_{c}=2 \pi c / 1025 \mathrm{~nm}$. This 
corresponds to an angular separation of $\mathrm{d} \lambda / \mathrm{d} y / 2=0.58 \mathrm{mrad}$ between consecutive attosecond bursts [31].

Because the target is located slightly outside the focal plane, the total angular separation includes another contribution arising from an interplay of pulse-front tilt and wave-front curvature [33]: The central lobe moves, on the time scale of one laser pulse, from a region with a wave-front pointing downwards to a region where it points upwards (compare Fig. 1). Our model predicts a total angular separation of 0.64 mrad including this effect (see Appendix D).

We demonstrate spatiotemporally coupled HHG using this setup by operating the cavity at the full seed power and supplying argon gas via a $100-\mu \mathrm{m}$-diameter end-fire nozzle placed $450 \mu \mathrm{m}$ in front of the focus. In general, a strong intracavity nonlinearity can affect the excited mode, especially at high finesse. However, for the parameters demonstrated here, no mode deformations were observed (Fig. 5(a)). To determine the cutoff energy, we coupled out the generated XUV radiation through a $207-\mu \mathrm{m}$-diameter hole, followed by two XUV/IR beam splitters and a 300-nm-thick aluminum filter, and analyzed it with a grating spectrometer (Fig. 5(b)). We observed harmonics up to $60 \mathrm{eV}$. For the measured pulse energy of $39 \mu \mathrm{J}$, our model predicts a peak intensity of $1.4 \times 10^{14} \mathrm{~W} / \mathrm{cm}^{2}$ in the target, corresponding to a high-harmonic cutoff energy of $59 \mathrm{eV}$.

To reach higher photon energies, we placed the target closer to the focus $(-150 \mu \mathrm{m})$ and used neon as target gas. As before, the gas plasma did not cause changes of the circulating mode (Fig. 5(c)). The XUV spectrum, measured through a 300-nm-thick zirconium filter, is shown in Fig. 5(d). The highest observed photon energies were around $120 \mathrm{eV}$, slightly below the expected cutoff of $155 \mathrm{eV}\left(4.2 \times 10^{14} \mathrm{~W} / \mathrm{cm}^{2}\right)$ resulting from the measured pulse energy of $57 \mu \mathrm{J}$, under the assumption of a Gaussian pulse shape. During the HHG measurements, we monitored the carrier-envelope offset phase of the seed employing a spectrally resolved $f$-to- $2 f$ interferometer [8]. Although not actively stabilized, the drift was below $300 \mathrm{mrad}$ over one measurement (50 s for argon, $203 \mathrm{~s}$ for neon).

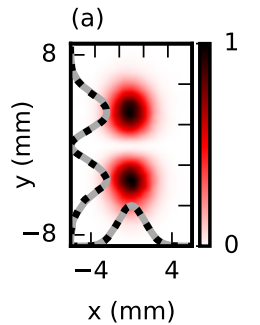

(c)

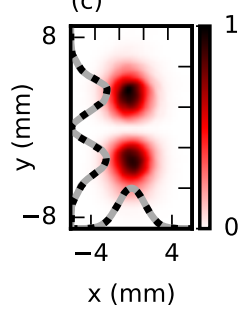

(b) argon, $z=-450 \mu \mathrm{m}(\mathrm{A})$

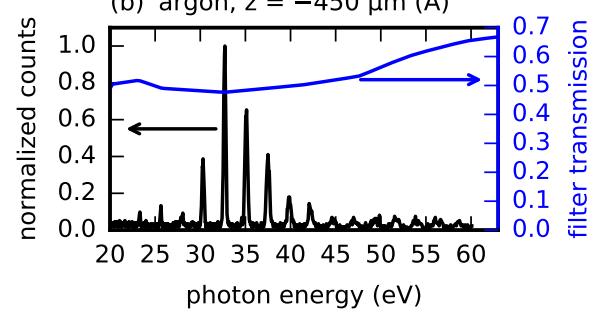

(d) neon, $z=-150 \mu \mathrm{m}$ (C)

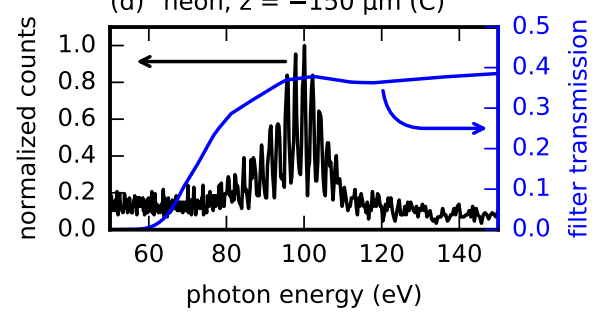

Fig. 5. (a) Transverse intensity profile of the cavity mode measured without target gas (color scale), integrated profiles in horizontal and vertical direction (gray lines), and integrated profiles with the target gas (black dotted lines). (b) Output-coupled high-harmonic spectrum (black) generated in an argon target placed $450 \mu \mathrm{m}$ in front of the focus, after transmission through a 300-nm Al filter (blue). (c,d) Same for a neon target placed $150 \mu \mathrm{m}$ in front of the focus, using a 300-nm $\mathrm{Zr}$ filter. 


\section{Discussion}

For the demonstrated experimental parameters, the angular separation predicted by our numerical model ( $0.68 \mathrm{mrad}$ in the case of argon, $0.61 \mathrm{mrad}$ for neon) is small compared to the divergence of the beamlets (argon: $6.1 \mathrm{mrad}$, neon:4.9 mrad), which leads to significant spatial overlap between the harmonic bursts, preventing the separation of isolated attosecond pulses and, thus, the observation of a spectral continuum. We compute an IAP contrast ratio $<1.1$ (compare Fig. 2(b); see also [30] and Appendix C). To achieve smaller divergence, it is necessary to operate at high photon energies and at the same time the nozzle must be placed far in front of the focus [16]. Therefore, more peak power is necessary, which can be either achieved by increasing the seed power or by using shorter pulses and a shorter delay (both within the limits given by the damage threshold of the mirror coatings). As a second measure to reduce the spatial overlap, the angular separation between the bursts can be increased. To this end, it is also beneficial to operate with shorter pulses. Our simulations (see Fig. 2(b)) have shown that 17.5-fs pulses and a delay of 2.5 cycles would be sufficient to produce IAPs around $95.6 \mathrm{eV}$ with a contrast ratio of $>20$ with the demonstrated geometry. For this, a pulse energy of $30 \mu \mathrm{J}$ is needed, leading to a peak intensity of only $0.63 \times 10^{10} \mathrm{~W} / \mathrm{cm}^{2}$ on the cavity mirrors and $3.0 \times 10^{14} \mathrm{~W} / \mathrm{cm}^{2}$ in a gas target placed $350 \mu \mathrm{m}$ in front of the focus. Although mirror coatings supporting pulses with a Fourier limit of $17.5 \mathrm{fs}$ in a four-mirror EC have already been demonstrated [25], it remains a technological challenge to achieve this value in a cavity with an optimum offset frequency of zero and more than four mirrors, mainly due to statistical errors in the coating procedure which make a large number of tries necessary.

\section{Conclusion}

In conclusion, we have demonstrated a femtosecond enhancement cavity with an intracavity wave-front rotation, enabled by the technique of transverse mode gating, and showed first HHG spectra of wave-form stable XUV pulse trains at photon energies beyond $100 \mathrm{eV}$ generated with this method, in a focusing/repetition-rate regime avoiding cumulative plasma effects [13,14] (see also Appendix G). Our experimental findings agree well with theoretical expectations. Numerical modeling shows that TMG can solve two main limitations of state-of-the art fs-EC-based XUV sources: First, the tradeoff between XUV generation efficiency and OC efficiency present with conventional hole OC can be circumvented thanks to an excellent OC efficiency irrespective of the target position, promising high-flux frequency combs in the XUV region for precision spectroscopy applications and, potentially, for future nuclear clocks [38]. Together with emerging $2-\mu \mathrm{m}$ technologies $[39,40]$, this method promises to boost the attainable photon energies of XUV combs to the water window and beyond. Secondly, it can be applied as an intracavity gating method to produce IAP at repetition rates in the tens of $\mathrm{MHz}$. When combined with ultrabroadband cavity mirror coatings and using a suitable step height, the demonstrated geometry and pulse energy are, according to our numerical model, already sufficient for IAP approaching $100 \mathrm{eV}$ with an excellent contrast ratio of $>20$.

\section{A. Numerical model}

The complex electric field $E$ of a pulse in a TEM $\mathrm{T}_{01}$ resonator mode can be written as

$$
E(x, y, z, \omega)=\widehat{\mathrm{TEM}}_{01}(x, y, z, \omega) E(\omega),
$$

where $x, y$ are the transverse coordinates, $z$ is the longitudinal coordinate, $\omega$ is the angular frequency, $E(\omega)$ describes the temporal dependence and $\widehat{\operatorname{TEM}}_{01}(x, y, z, \omega)$ the spatial dependence. For a Gaussian pulse, $E(\omega)$ is the Fourier transform of $E(t)=A(t) \exp \left(i \omega_{c} t\right)$, with the envelope $A(t)=\exp \left(-t^{2} / \tau^{2}\right)$, central frequency $\omega_{c}$, and $\tau=t_{\mathrm{FWHM}} / \sqrt{\log 4}$ with the full-width-half- 
maximum intensity pulse duration $t_{\mathrm{FWHM}}$. The formulas for the normalized mode $\widehat{\mathrm{TEM}}_{01}$ are provided in [41, p. 645].

To introduce spatiotemporal coupling, we write down this field in a far-field plane $\left(z_{\mathrm{ff}}=-10^{5} z_{R}\right.$, with the Rayleigh range $z_{R}=\sqrt{z_{R, x} \cdot z_{R, y}}$ ) and apply a delay $\Delta t$ to the lower lobe:

$$
\begin{aligned}
& E_{\mathrm{TMG}}\left(x, y, z_{\mathrm{ff}}, \omega\right)= \\
& \quad=E\left(x, y, z_{\mathrm{ff}}, \omega\right) \exp (-i \omega \Delta t H(-y))
\end{aligned}
$$

Here, $H(\cdot)$ is the Heaviside step function. To calculate the XUV divergence and the angular separation of the harmonic bursts, we need the driving field in the target plane. For this, we numerically computed $E_{\mathrm{TMG}}\left(x, y, z_{\mathrm{ff}}, \omega\right)$ on a uniformly spaced $(x, y, \omega)$ grid centered around $\left(0,0, \omega_{c}\right)$ and propagated the field to the target position $z_{t}$, using a Fresnel two-step propagator [42, Appendix B]. Then, we applied a discrete Fourier transform to obtain the complex envelope $E_{\mathrm{TMG}}\left(x, y, z_{\mathrm{t}}, t\right)$ on a $(x, y, t)$ grid.

Due to the strong nonlinearity of the high-harmonic generation (HHG) process, the main contribution to the XUV emission stems from the region around the maximum intensity $c \epsilon_{0} / 2\left|E_{\mathrm{TMG}}\left(x, y, z_{\mathrm{t}}, t\right)\right|^{2}$, located within the central lobe. In the vincinity of this maximum, we can approximate the field by a vertically tilted fundamental Gaussian beam:

$$
\begin{aligned}
& E_{\mathrm{TMG}}\left(x, y, z_{\mathrm{t}}, t_{\mathrm{max}}\right) \approx \\
& \quad \approx C \exp \left(-i k\left(\frac{x_{r}^{2}}{2 q_{x}}+\frac{y_{r}^{2}}{2 q_{y}}\right)-i k \beta_{y} y_{r}\right)
\end{aligned}
$$

with $x_{r}=x-x_{\max }, y_{r}=y-y_{\max }, k=\omega_{c} / c$, the complex beam parameters $q_{x}$ and $q_{y}$, the vertical wave-front direction $\beta_{y}$ and

$$
\left(x_{\max }, y_{\max }, t_{\max }\right)=\underset{(x, y, t)}{\arg \max }\left|E_{\mathrm{TMG}}\left(x, y, z_{\mathrm{t}}, t\right)\right|^{2} .
$$

We determined the parameters $q_{x}, q_{y}, \beta_{y}$ numerically with fitting procedures for the amplitude and phase along $x$ and $y$ line cuts through the intensity maximum, discarding data points where the intensity was below half the maximum. This resulted in effective driving beam radii $w_{x, \text { eff }}=\sqrt{2 /\left|\mathfrak{I}\left(k q_{x}^{-1}\right)\right|}, w_{y, \text { eff }}=\sqrt{2 /\left|\mathfrak{I}\left(k q_{y}^{-1}\right)\right|}$ and wave-front curvatures $R_{x, \text { eff }}^{-1}=\mathfrak{R}\left(q_{x}^{-1}\right)$, $R_{y, \text { eff }}^{-1}=\mathfrak{R}\left(q_{y}^{-1}\right)$ in the plane of the gas target. We then approximated the spot sizes $w_{H}$ and wave-front curvatures $R_{H}^{-1}$ of the XUV beam at $z_{t}$ in $y$ direction by assuming a power law for the driving-intensity dependence of the single-atom dipole amplitude and a linear relationship for the single-atom dipole phase [16, Appendix C]:

$$
\begin{gathered}
w_{H}=w_{\mathrm{eff}} / \sqrt{N_{H}} \\
R_{H}^{-1}=R_{\mathrm{eff}}^{-1}+\frac{4 c \alpha_{H} I_{t}}{H \omega_{c} w_{\mathrm{eff}}^{2}}
\end{gathered}
$$

Here, $N_{H}$ and $\alpha_{H}$ are coefficients describing the driving-intensity dependence of the singleatom dipole amplitude and phase, respectively, $H$ is the harmonic order and $I_{t}$ is the peak driving intensity in the plane of the gas target. The divergence of a beam with a near-field spot radius $w_{H}$, wave-front curvature $R_{H}^{-1}$ and frequency $H \omega_{c}$ is (compare [16])

$$
\theta=\sqrt{\frac{w_{H}^{2}}{R_{H}^{2}}+\frac{4 c^{2}}{H^{2} \omega_{c}^{2} w_{H}^{2}}} .
$$


In the experiment, the position of the delay mirror relative to the focus was different from the value $z_{\mathrm{ff}}=-10^{5} z_{R}$ used here (in particular, the delay mirror was placed behind a focusing mirror). However, the exact position is irrelevant as long as the delay mirror is imaged onto a plane that is far away from the focus, which was accomplished by placing the delay mirrors at a distance of roughly $R=300 \mathrm{~mm}$ from the focusing mirrors [26, Appendix A].

To determine the output coupling efficiency for the parameters considered in the main text $\left(t_{\mathrm{FWHM}}=40 \mathrm{fs}, \omega_{c}=c / 1025 \mathrm{~nm}\right.$, a delay $\Delta t$ of 0.5 cycles and values for $q_{x}, q_{y}$ corresponding to a focal spot size of $w_{0, x} \times w_{0, y}=17.2 \times 11.8 \mu \mathrm{m}^{2}$ ), we computed the effective beam radius

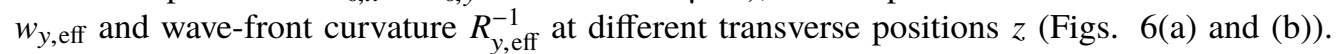
Then, the near-field XUV beam radius and wave-front curvature was calculated according to (5) and (6) for the two cases of intermediate (argon, $H=33, I_{t}=1.5 \times 10^{14} \mathrm{~W} / \mathrm{cm}^{2}$ ) and high (neon, $H=79, I_{t}=1.5 \times 10^{14} \mathrm{~W} / \mathrm{cm}^{2}$ ) photon energy generation (Figs. 6(c) and (d)). For this, we used intensity-dependence parameters $N_{H}, \alpha_{H}$ taken from [16, Table B1]. The pulse energies necessary to achieve the respective peak intensities $I_{t}$ in the target (Fig. 6(e)) were calculated numerically by integrating $I\left(x, y, z_{\mathrm{t}}, t\right)=c \epsilon_{0} / 2\left|E_{\mathrm{TMG}}\left(x, y, z_{\mathrm{t}}, t\right)\right|^{2}$ over the $(x, y, t)$ grid after normalization to a peak intensity of $I_{t}$. Applying Eq. (7) then yields the expected divergence of the XUV radiation in $y$ direction (Fig. 6(f)). As expected, the smallest divergence is obtained when the gas target is located in front of the focal plane [16,34].

The XUV output coupling efficiency attainable by employing an mirror with an on-axis slit opening depends on the angular width $\delta=d / z_{m}$ of the slit, where $d$ is the slit width and $z_{m}$ the distance of the output coupling mirror from the focus. This angular width also determines the round-trip losses that the circulating mode experiences at the output coupling mirror. To find an acceptable value, we calculated the round-trip loss for different slit angular widths $\delta$. To this end, we numerically computed the ratio of the energy lost by transmission through the slit and the total energy incident on the output coupling mirror (far-field beam profile given by Eq. (2)). The round-trip loss is twice the resulting value, because the same amount of energy that is transmitted through the slit is also scattered to nonresonant higher-order modes [6]:

$$
L(\delta)=2\left(\frac{\iiint_{y<\delta z_{\mathrm{ff}} / 2}\left|E_{\mathrm{TMG}}\left(x, y, z_{\mathrm{ff}}, \omega\right)\right|^{2} \mathrm{~d} x \mathrm{~d} y \mathrm{~d} \omega}{\iiint\left|E_{\mathrm{TMG}}\left(x, y, z_{\mathrm{ff}}, \omega\right)\right|^{2} \mathrm{~d} x \mathrm{~d} y \mathrm{~d} \omega}\right)
$$

Figure 6(g) shows the resulting losses versus slit angular width. To keep the losses below $1 \%$, a value consistent with the typical finesse aimed for in cavity-enhanced HHG experiments, we chose picked an angular width of $7.31 \mathrm{mrad}$.

Then, we computed the output coupling efficiency $\epsilon$, again by spatial integration. For this, we took advantage of the approximation of the harmonic beam profile as a Gaussian $\left|E_{\mathrm{XUV}}(x, y)\right|^{2} \propto \exp \left(-2 y^{2} / w_{m, y}^{2}\right) \exp \left(-2 x^{2} / w_{m, x}^{2}\right)[16]$, where $w_{m, x}$ and $w_{m, y}=\theta z_{m}$ are the XUV beam radii in $x$ and $y$ direction on the output coupling mirror:

$$
\epsilon=\frac{\iint_{y<\delta z_{m} / 2}\left|E_{\mathrm{XUV}}(x, y)\right|^{2} \mathrm{~d} x \mathrm{~d} y}{\iint\left|E_{\mathrm{XUV}}(x, y)\right|^{2} \mathrm{~d} x \mathrm{~d} y}=\operatorname{erf}(\delta / \theta / \sqrt{2})
$$

This resulted in output coupling efficiencies in the range $40-100 \%$ for all considered gas target positions (Fig. 6(h)).

The delay of one half cycle, necessary to obtain an on-axis intensity maximum in the focus region, introduces a slight wave-front rotation and thus angular separation between consecutive bursts. To validate that efficient output coupling of all bursts is still possible, we computed the angular separation (compare following sections) for the cases of intermediate (H33 in argon) and high (H79 in neon) photon energy production and obtained values $<0.17 \mathrm{mrad}$ for all considered gas target positions, which is well below the computed divergence (compare Fig. 6(f)) and the assumed slit angular width of $7.31 \mathrm{mrad}$. 


\section{Optics EXPRESS}
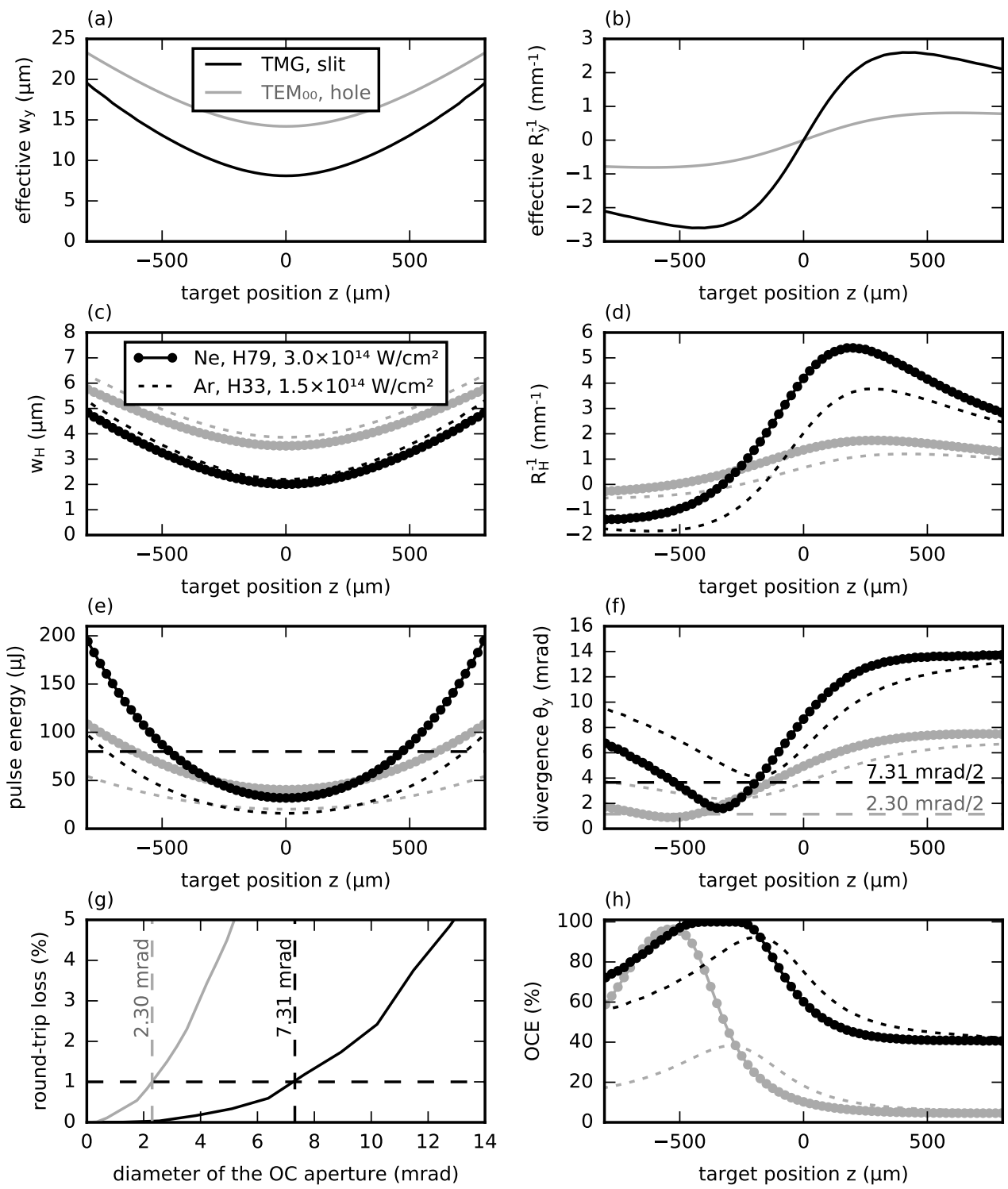

Fig. 6. a) Beam radius in $y$ direction of a fundamental Gaussian mode fitted to the central lobe of the simulated TMG mode (delay 0.5 cycles, black) and of the fundamental Gaussian mode with the same complex beam parameter for comparison (gray). b) Wave-front curvature for both cases. c,d) Harmonic beam radius and wave-front curvature in $y$ direction, calculated in the plane of the gas target with a simple analytical single-trajectory model for the harmonic dipole response, for generation parameters allowing for high photon energy (solid line with dot markers) and intermediate photon energy (dashed line). e) Pulse energy needed to reach $3 \times 10^{14} \mathrm{~W} / \mathrm{cm}^{2}$ (high photon energy case) and $1.5 \times 10^{14} \mathrm{~W} / \mathrm{cm}^{2}$ (intermediate photon energy case) in the gas target plane with the TMG mode (black) and the fundamental Gaussian mode (gray). f) Harmonic beam divergence in $y$ direction, resulting from its beam radius and wave-front curvature in the gas target plane. g) Round-trip losses of the simulated TMG mode due to an on-axis output coupling slit with given angular width (black), and of the fundamental Gaussian mode due to an on-axis hole with given angular diameter (gray). h) XUV output coupling efficiencies resulting from the computed divergences, assuming output coupling apertures with a round-trip loss of $1 \%$. 


\section{B. Comparison with output coupling using the fundamental mode}

For comparison, we repeated the same procedure to determine the output coupling efficiencies attainable with the fundamental Gaussian mode and a circular opening in the output coupling mirror. For this, we used the same numerical model but replaced the $\mathrm{TEM}_{01}$ mode by a symmetric $\mathrm{TEM}_{00}$ mode with the same complex beam paramters and set $\Delta t=0$. As before, we computed the round-trip losses numerically by spatial integration, but this time assuming a circular aperture:

$$
L(\delta)=2\left(\frac{\iiint_{\sqrt{x^{2}+y^{2}}<\delta z_{\mathrm{ff}} / 2}|E|^{2} \mathrm{~d} x \mathrm{~d} y \mathrm{~d} \omega}{\iiint|E|^{2} \mathrm{~d} x \mathrm{~d} y \mathrm{~d} \omega}\right) .
$$

The output coupling efficiency with a hole angular diameter $\delta=2.30 \mathrm{mrad}$ for $1 \%$ round-trip losses was computed analytically as

$$
\begin{gathered}
\epsilon=\frac{\iint_{\sqrt{x^{2}+y^{2}}<\delta z_{m} / 2}\left|E_{\mathrm{XUV}}\right|^{2} \mathrm{~d} x \mathrm{~d} y}{\iint\left|E_{\mathrm{XUV}}\right|^{2} \mathrm{~d} x \mathrm{~d} y}= \\
=1-\exp \left(-2(\delta / \theta / \sqrt{2})^{2}\right)
\end{gathered}
$$

The dependence of the resulting quantities on the gas target position is shown in Fig. 6 in gray.

\section{Gating efficiency}

To estimate the angular separation between consecutive attosecond bursts, we compared the wavefront direction $\beta_{y}$ at $t_{\max }$ with the wave-front direction $\beta_{y}^{\prime}$ one half cycle later $\left(t^{\prime}=t_{\max }+T / 2\right.$, with $\left.T=2 \pi / \omega_{c}\right)$, which was determined similarly to $\beta_{y}$ by approximating the driving field in the vicinity of the intensity maximum by a fundamental Gaussian beam with vertically tilted phase fronts:

$$
\begin{aligned}
& E_{\mathrm{TMG}}\left(x, y, z_{\mathrm{t}}, t^{\prime}\right) \approx \\
& \quad \approx C \exp \left(-i k\left(\frac{x_{r}^{\prime 2}}{2 q_{x}^{\prime}}+\frac{y_{r}^{\prime 2}}{2 q_{y}^{\prime}}\right)-i k \beta_{y}^{\prime} y_{r}^{\prime}\right)
\end{aligned}
$$
and

and fitting the parameters $q_{x}^{\prime}, q_{y}^{\prime}$ and $\beta_{y}^{\prime}$ along $x / y$ line cuts, with $x_{r}^{\prime}=x-x_{\max }^{\prime}, y_{r}^{\prime}=y-y_{\max }^{\prime}$

$$
\left(x_{\max }^{\prime}, y_{\max }^{\prime}\right)=\underset{(x, y)}{\arg \max }\left|E_{\mathrm{TMG}}\left(x, y, z_{\mathrm{t}}, t^{\prime}\right)\right|^{2} .
$$

This allowed us to approximate the angular separation $\Delta \beta=\beta_{y}^{\prime}-\beta_{y}$ between the XUV beamlets of consecutive half cycles. Assuming an infinitesimally thin output coupling slit placed in the direction $\beta_{y}$ of the strongest beamlet, the output coupled isolated attosecond pulse will have satellite pulses stemming from the XUV emission of neighboring half cycles due to spatial overlap [30, Supplementary Information, section 3.2]. The intensity of the main pulse relative to these satellite pulses, i.e., the contrast ratio $\Gamma$ of the isolated attosecond pulse, is given by the divergence $\theta$ of the beamlets:

$$
\Gamma=1 /\left|\exp \left(-\Delta \beta^{2} / \theta^{2}\right)\right|^{2}
$$

Figure 7 shows the dependence of the quantities $\theta, \Delta \beta$ and $\Gamma$ on the target position for the case of isolated attosecond pulse generation using 17.5 -fs pulses in neon and for the experimentally demonstrated parameters. 

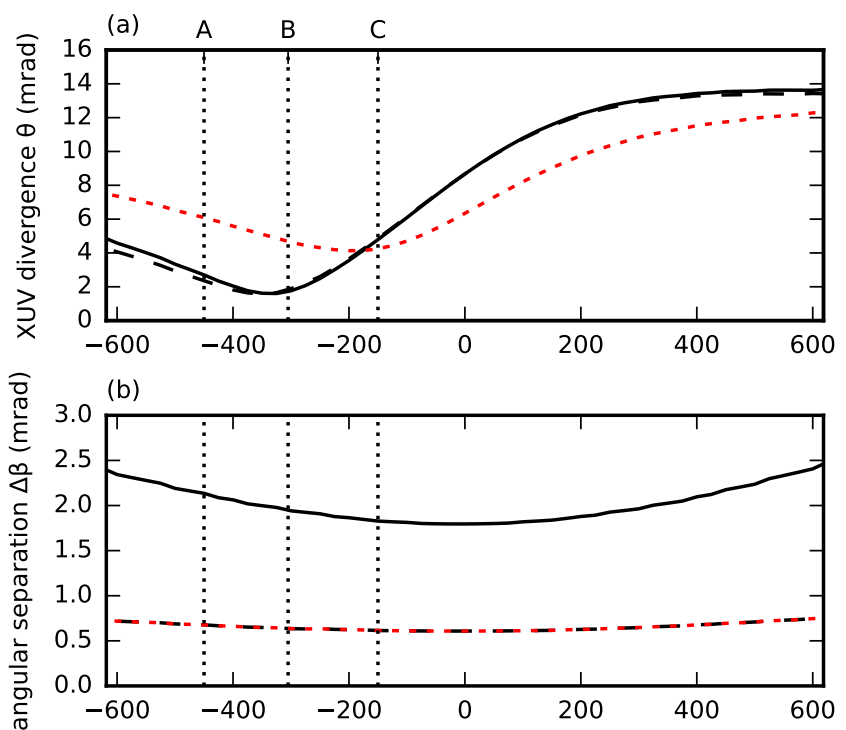

(c)

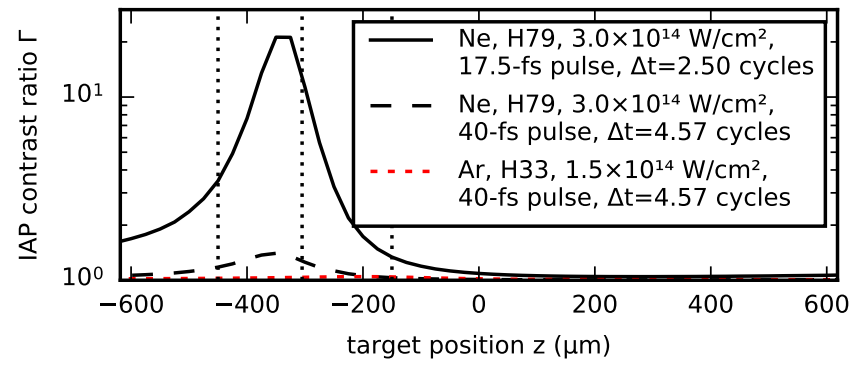

Fig. 7. a) XUV divergence in $y$ direction for parameters enabling the gating of isolated attosecond pulses (solid line; see legend in (c)) and for the experimentally demonstrated parameters (dashed lines). b) Angular separation between consecutive attosecond bursts according to the numerical model. c) Resulting contrast ratio between strongest attosecond burst and neighboring bursts. The vertical dotted lines labeled with $\mathrm{A}, \mathrm{B}$ and $\mathrm{C}$ mark the $z$ position for the data shown in Figs. 5(b), 4 and 5(d), respectively.

\section{Contributions to the angular separation}

The total angular separation comprises contributions from the spatial chirp and, outside the focal plane, from the interplay of pulse front tilt and wave-front curvature, as illustrated in [33]. In the main text, we showed how to calculate the first contribution $\Delta \beta_{c}=-\pi c \omega_{c}^{-2} \gamma /\left(\gamma^{2}+\left(w_{y} / \Delta \omega\right)^{2}\right)$ from the spatial dispersion $\gamma$, the the $1 / e^{2}$-intensity spectral width $\Delta \omega$ and the lobe radius $w_{y}$. Here, we describe how the contribution $\Delta \beta_{t}$ from the pulse front tilt can be quantified.

As can be seen in Fig. 1 of the main text, the delay between the two lobes leads to a pulse front tilt around the focus region: the intensity maximum of the central lobe moves, on the time scale of one laser pulse, along the vertical direction $y$. Due to the wave-front curvature $R_{y \text {, fff }}^{-1}$, the wave-front direction $\beta_{t}$ varies along this coordinate with a constant rate $\mathrm{d} \beta_{t} / \mathrm{d} y=-R_{y, \text { eff }}^{-1}$. For a given vertical velocity $v$ of the intensity maximum, the vertical distance between the emission sites of two consecutive bursts is $v T / 2$. Their angular separation is thus

$$
\Delta \beta_{t}=v T / 2 R_{y, \text { eff }}^{-1}
$$




\section{Optics EXPRESS}

At each target position $z_{t}$, we calculated both contributions assuming the parameters for isolated attosecond pulse generation (compare Fig. 7(c), solid line). For this, we computed the spatial dispersion by performing a linear fit to the function $\omega \mapsto \arg \max _{y}\left|E_{\mathrm{TMG}}\left(x_{\max }, y, z_{\mathrm{t}}, \omega\right)\right|^{2}$, evaluated along the $(y, \omega)$ grid. The lobe radius $w_{y, \text { eff }}$ and wave-front curvature $R_{y, \text { eff }}^{-1}$ were determined as described before, and the spectral width of a Gaussian pulse is $\Delta \omega=2 \sqrt{\log 4} / t_{\text {FWHM }}$. A linear fit to the function $t \mapsto \arg \max _{y}\left|E_{\mathrm{TMG}}\left(x_{\max }, y, z_{\mathrm{t}}, t\right)\right|^{2}$, evaluated along the $(y, t)$ grid, was used to determine the vertical velocity $v$ of the intensity maximum. Figure 8 shows that this simple semi-analytical model for the two contributions $\Delta \beta_{c}$ and $\Delta \beta_{t}$ achieves acceptable agreement with the numerically determined total angular separation $\Delta \beta$.

The spatial dispersion was computed in the same way for the TMG mode used in the experiment (40-fs pulse, $\Delta t=4.57 T)$. At the position where the transverse mode was imaged $(z=-305 \mu \mathrm{m})$, we obtained a value of $-53 \mu \mathrm{m} / \mathrm{PHz}$.
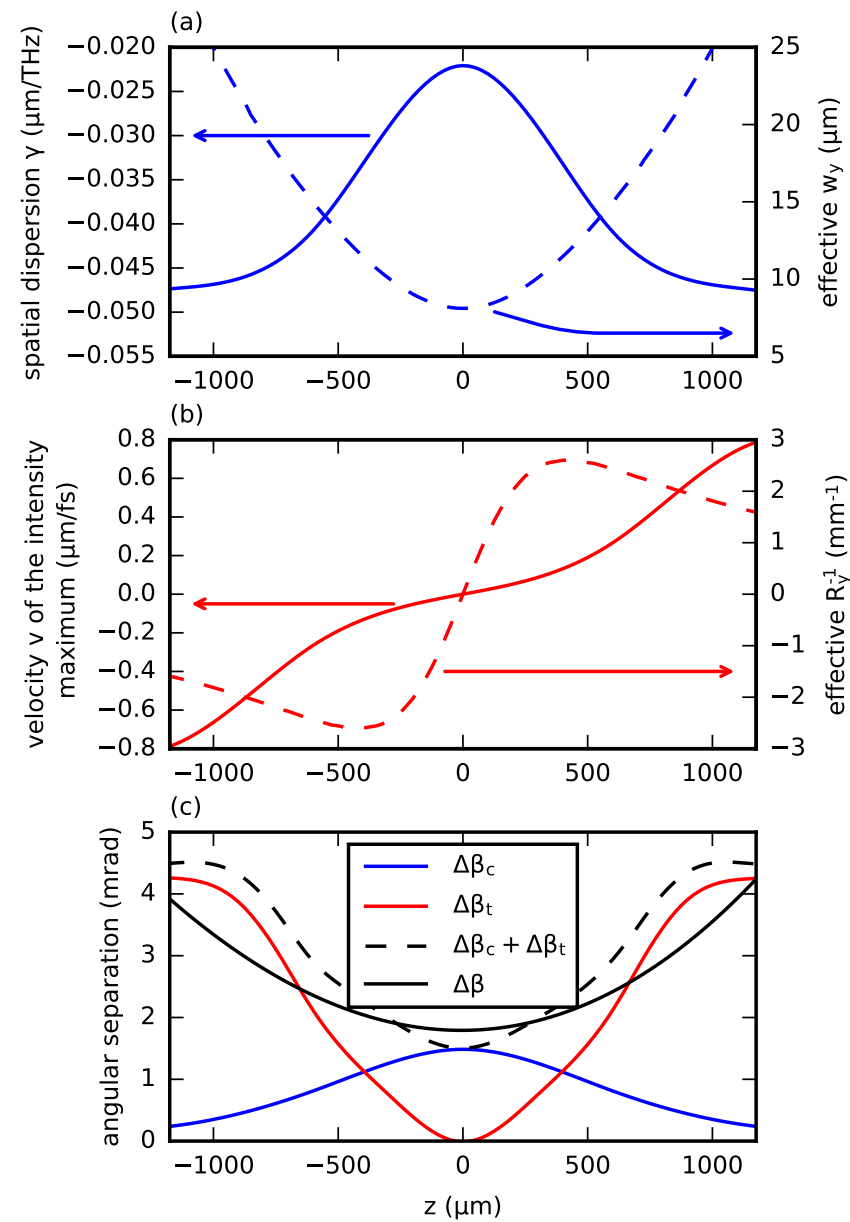

Fig. 8. a) Relevant parameters $\gamma, w_{y}$ for the spatial chirp contribution to the angular separation, for parameters enabling the production of isolated attosecond pulses (compare Fig. 7(c), solid line). b) The same for the contribution from the pulse-front tilt. c) Semianalytical approximations $\Delta \beta_{c}=-\pi c \omega_{c}^{-2} \gamma /\left(\gamma^{2}+\left(w_{y} / \Delta \omega\right)^{2}\right)$ (spatial chirp contribution to the angular separation), $\Delta \beta_{t}=-v T / 2 R_{y}^{-1}$ (pulse-front tilt contribution) and their sum, compared to the numerically computed value $\Delta \beta$. 


\section{E. Calibration of the $z$ position in the experiments}

\section{E.1. Imaging the spatial dispersion}

The focal region was imaged via a silicon nitride plate and two lenses. To determine the magnification factor and the $z$ coordinate of the imaged plane for the data shown in Fig. 4 of the main text, we measured the distance between the two lenses $(182 \mathrm{~cm})$. Because it would be difficult to measure distances between the first lens (focal length $f=50 \mathrm{~mm}$ ) and the focus and between the second lens $(f=200 \mathrm{~mm})$ and the camera with sufficient precision, we recorded the caustic of the beam after the second lens by translating the camera on a stage. From this, we obtained the vertical divergence of the corresponding beam. The vertical divergence of the beam before the first lens can be calculated from the beam waist $w_{0, y}=11.8 \mu \mathrm{m}$, resulting from the mode size on the mirror $\left(w_{y}=4.16 \mathrm{~mm}\right)$ and the radius of curvature of the focusing mirrors $(R=300 \mathrm{~mm})$. Knowing the divergence of the beam after the second and before the first lens, we selected beam radii on the two lenses consistent with these divergences by solving the corresponding system of two equations. Because the beam between both lenses is fully determined by these radii, this allows us to calculate the distances between the first lens and the focus and between the second lens and the camera, resulting in a magnification factor $U=-2.16$ and position $z=-305 \mu \mathrm{m}$ for the plane imaged in Fig. 4 of the main text.

\section{E.2. Position of the gas nozzle}

The position $z$ of the gas target relative to the focus was determined by translating the nozzle along the $z$ axis while observing the plasma glow. We assumed that the position of the focus is in the middle of the two points where the plasma glow vanishes.

\section{F. Calibration of the circulating power}

The circulating power was calculated by integrating the intracavity power spectral density, which was determined indirectly by recording the power and spectrum transmitted through one of the plane, highly reflective cavity mirrors. We accounted for the spectrally dependent transmission curve of this mirror, which was measured with a spectrophotometer and calibrated using the power before and after the mirror while illuminated with a known spectrum. This approach allowed us to obtain correct values for the circulating power even in the case of strong ionization-induced spectral blueshift. For the empty cavity, we measured values up to $1.5 \mathrm{~kW}$, corresponding to a pulse energy of $80 \mu \mathrm{J}$.

\section{G. Cumulative plasma effects}

The velocity of the gas ejected by the 100- $\mu$ m-diameter nozzle, pointing in the $x$ direction, was computed with the analytical model described in [43, p. 17ff.], presuming a reservoir temperature of $300 \mathrm{~K}$ and a backing pressure in the order of 10 bar. This resulted in velocities $>280 \mathrm{~m} / \mathrm{s}$ for $\operatorname{argon}$ and $>390 \mathrm{~m} / \mathrm{s}$ for neon gas. At a repetition rate of $18.4 \mathrm{MHz}$, this corresponds to a translation distances of $15.2 \mu \mathrm{m}$ (argon) and $21.2 \mu \mathrm{m}$ (neon) between consecutive pulses. For comparison, the diameter $2 w_{x, H}=2 w_{x, \text { eff }} / \sqrt{N_{H}}$ over which harmonics are created was $<10.7 \mu \mathrm{m}$ for argon and $<9.7 \mu \mathrm{m}$ for neon, for gas target positions $|z|<500 \mu \mathrm{m}$. Consequently, the demonstrated setup operated in a regime where the target gas is fully replenished between pulses so that the build-up of a cumulative plasma can be avoided.

\section{H. Mode matching}

In theory, the overlap of a Gaussian seeding mode with a spatial offset and a $\mathrm{TEM}_{01}$ mode can be $47.8 \%$ (value calculated with the numerical model). We measured a mode matching efficiency of only $30 \%$, which can be attributed to several factors: First, the ellipticity of the seeding mode 
did not perfectly match the ellipticity of the respective lobe of the cavity mode, second, the seeding mode was not a perfect Gaussian mode due to the compression scheme, and third, the measured overlap also includes the spectral overlap. The overlap can be improved by shaping the seeding beam using cylindrical lenses and a phase mask, so that, in theory, a value of $82.7 \%$ is possible [44]. Even better coupling efficiency can be achieved by using an input coupler with a half-sided highly-reflective coating [27], reaching values up to 98.2\%. Alternatively, intracavity wave-front rotation could be obtained with a fundamental mode by placing transmissive or reflective wedges inside the resonator [45].

\section{Comparison to the quasi-imaging method}

Quasi-imaging-based output coupling methods require the operation of the resonator precisely in the middle of its stability range [20]. This imposes a limitation on the maximum beam radius $w_{m}=\sqrt{\lambda /\left|\pi \mathfrak{J}\left(q^{-1}\right)\right|}$ on the focusing mirrors, determined in first approximation only by the cavity length $L$ and the wavelength $\lambda$ [20, equation 17]:

$$
w_{m}=\sqrt{\frac{L \lambda}{\pi}}
$$

For the experimental parameters $L=16.3 \mathrm{~m}, \lambda=1025 \mathrm{~nm}$, this results in a maximum beam radius of $2.3 \mathrm{~mm}$. Using the same approach as described before, we numerically computed the electric far-field of the symmetric quasi-imaging mode $\sqrt{3 / 11} \widehat{\mathrm{TEM}}_{00}-\sqrt{8 / 11} \widehat{\mathrm{TEM}}_{04}$ (compare [21]) with this radius on a $(x, y, \omega)$ grid, for a $40-\mathrm{fs}$ Gaussian pulse, and applied a discrete Fourier transform along the $\omega$ axis to obtain the complex envelope $E_{\mathrm{QI}}(x, y, t)$. The ratio of the peak intensity $\max \left[c \epsilon_{0} / 2\left|E_{\mathrm{QI}}\left(x, y, z_{\mathrm{ff}}, t\right)\right|^{2}\right]$ and the peak power, obtained by spatial integration, was $8.55 \mathrm{~cm}^{-2}$. For a damage threshold of $1 \times 10^{10} \mathrm{~W} / \mathrm{cm}^{2}$, the maximum permitted peak power is therefore $1 \times 10^{10} \mathrm{~W} / \mathrm{cm}^{2} /\left(8.55 \mathrm{~cm}^{-2}\right)=1.17 \mathrm{GW}$. This value scales linearly with $L$, i.e., a shorter cavity permits less peak power when utilizing a quasi-imaging mode.

This limitation does not apply to output coupling with the TMG mode because operation in the middle of the stability range is not necessary. In our setup, the focusing mirrors were placed at a distance of $150 \mathrm{~mm}$ from the focus. The calculated peak intensity on the mirrors was $7.4 \times 10^{9} \mathrm{~W} / \mathrm{cm}^{2}$ for a pulse energy of $80 \mu \mathrm{J}$, corresponding to a peak power of $1.88 \mathrm{GW}$ for 40-fs pulses.

\section{Funding}

MEGAS Fraunhofer/Max-Planck-Gesellschaft cooperation.

\section{References}

1. C. Gohle, T. Udem, M. Herrmann, J. Rauschenberger, R. Holzwarth, H. A. Schuessler, F. Krausz, and T. W. Hänsch, "A frequency comb in the extreme ultraviolet," Nature 436, 234-237 (2005).

2. R. Jones, K. Moll, M. Thorpe, and J. Ye, "Phase-Coherent Frequency Combs in the Vacuum Ultraviolet via High-Harmonic Generation inside a Femtosecond Enhancement Cavity,” Phys. Rev. Lett. 94, 193201 (2005).

3. A. Cingöz, D. C. Yost, T. K. Allison, A. Ruehl, M. E. Fermann, I. Hartl, and J. Ye, "Direct frequency comb spectroscopy in the extreme ultraviolet," Nature 482, 68-71 (2012).

4. C. Benko, T. K. Allison, A. Cingöz, L. Hua, F. Labaye, D. C. Yost, and J. Ye, "Extreme ultraviolet radiation with coherence time greater than $1 \mathrm{~s}$, , Nat. Photonics 8, 530-536 (2014).

5. M. Herrmann, M. Haas, U. D. Jentschura, F. Kottmann, D. Leibfried, G. Saathoff, C. Gohle, A. Ozawa, V. Batteiger, S. Knünz, N. Kolachevsky, H. A. Schüssler, T. W. Hänsch, and T. Udem, "Feasibility of coherent xuv spectroscopy on the 1 S - 2 S transition in singly ionized helium," Phys. Rev. A 79, 052505 (2009).

6. I. Pupeza, S. Holzberger, T. Eidam, H. Carstens, D. Esser, J. Weitenberg, P. Rußbüldt, J. Rauschenberger, J. Limpert, T. Udem, A. Tünnermann, T. W. Hänsch, A. Apolonski, F. Krausz, and E. Fill, "Compact high-repetition-rate source of coherent $100 \mathrm{eV}$ radiation," Nat. Photonics 7, 608-612 (2013). 
7. H. Carstens, M. Högner, T. Saule, S. Holzberger, N. Lilienfein, A. Guggenmos, C. Jocher, T. Eidam, D. Esser, V. Tosa, V. Pervak, J. Limpert, A. Tünnermann, U. Kleineberg, F. Krausz, and I. Pupeza, "High-harmonic generation at 250 MHz with photon energies exceeding $100 \mathrm{eV}$," Optica 3, 366 (2016).

8. T. Saule, S. Heinrich, J. Schötz, N. Lilienfein, M. Högner, O. deVries, M. Plötner, J. Weitenberg, D. Esser, J. Schulte, P. Russbueldt, J. Limpert, M. F. Kling, U. Kleineberg, and I. Pupeza, "High-flux ultrafast extreme-ultraviolet photoemission spectroscopy at $18.4 \mathrm{MHz}$ pulse repetition rate," Nat. Commun. 10, 458 (2019).

9. C. Corder, P. Zhao, J. Bakalis, X. Li, M. D. Kershis, A. R. Muraca, M. G. White, and T. K. Allison, "Ultrafast extreme ultraviolet photoemission without space charge," Struct. Dyn. 5, 054301 (2018).

10. D. R. Carlson, J. Lee, J. Mongelli, E. M. Wright, and R. J. Jones, "Intracavity ionization and pulse formation in femtosecond enhancement cavities," Opt. Lett. 36, 2991-2993 (2011).

11. T. K. Allison, A. Cingöz, D. C. Yost, and J. Ye, "Extreme Nonlinear Optics in a Femtosecond Enhancement Cavity," Phys. Rev. Lett. 107, 183903 (2011).

12. S. Holzberger, N. Lilienfein, H. Carstens, T. Saule, M. Högner, F. Lücking, M. Trubetskov, V. Pervak, T. Eidam, J. Limpert, A. Tünnermann, E. Fill, F. Krausz, and I. Pupeza, "Femtosecond Enhancement Cavities in the Nonlinear Regime," Phys. Rev. Lett. 115, 023902 (2015).

13. G. Porat, C. M. Heyl, S. B. Schoun, C. Benko, N. Dörre, K. L. Corwin, and J. Ye, "Phase-matched extreme-ultraviolet frequency-comb generation," Nat. Photonics 12, 387-391 (2018).

14. T. Saule, M. Högner, N. Lilienfein, O. de Vries, M. Plötner, V. S. Yakovlev, N. Karpowicz, J. Limpert, and I. Pupeza, "Cumulative plasma effects in cavity-enhanced high-order harmonic generation in gases," APL Photonics 3, 101301 (2018).

15. N. Lilienfein, C. Hofer, M. Högner, T. Saule, M. Trubetskov, V. Pervak, E. Fill, C. Riek, A. Leitenstorfer, J. Limpert, F. Krausz, and I. Pupeza, "Temporal solitons in free-space femtosecond enhancement cavities," Nat. Photonics 13, 214-218 (2019).

16. M. Högner, T. Saule, and I. Pupeza, "Efficiency of cavity-enhanced high harmonic generation with geometric output coupling,” J. Phys. B: At. Mol. Opt. Phys. 52, 075401 (2019).

17. K. D. Moll, R. J. Jones, and J. Ye, "Output coupling methods for cavity-based high-harmonic generation," Opt. Express 14, 8189-8197 (2006).

18. J. Wu and H. Zeng, "Cavity-enhanced noncollinear high-harmonic generation for extreme ultraviolet frequency combs," Opt. Lett. 32, 3315-3317 (2007).

19. A. Ozawa, A. Vernaleken, W. Schneider, I. Gotlibovych, T. Udem, and T. W. Hänsch, "Non-collinear high harmonic generation: a promising outcoupling method for cavity-assisted XUV generation," Opt. Express 16, 6233-6239 (2008).

20. J. Weitenberg, P. Rußbüldt, T. Eidam, and I. Pupeza, "Transverse mode tailoring in a quasi-imaging high-finesse femtosecond enhancement cavity," Opt. Express 19, 9551-9561 (2011).

21. I. Pupeza, M. Högner, J. Weitenberg, S. Holzberger, D. Esser, T. Eidam, J. Limpert, A. Tünnermann, E. Fill, and V. Yakovlev, "Cavity-Enhanced High-Harmonic Generation with Spatially Tailored Driving Fields," Phys. Rev. Lett. 112, 103902 (2014).

22. M. I. Stockman, M. F. Kling, U. Kleineberg, and F. Krausz, “Attosecond nanoplasmonic-field microscope,” Nat. Photonics 1, 539-544 (2007)

23. B. Förg, J. Schötz, F. Süßmann, M. Förster, M. Krüger, B. Ahn, W. A. Okell, K. Wintersperger, S. Zherebtsov, A. Guggenmos, V. Pervak, A. Kessel, S. A. Trushin, A. M. Azzeer, M. I. Stockman, D. Kim, F. Krausz, P. Hommelhoff, and M. F. Kling, "Attosecond nanoscale near-field sampling," Nat. Commun. 7, 11717 (2016).

24. J. Schötz, B. Förg, M. Förster, W. A. Okell, M. I. Stockman, F. Krausz, P. Hommelhoff, and M. F. Kling, "Reconstruction of Nanoscale Near Fields by Attosecond Streaking," IEEE J. Sel. Top. Quantum Electron. 23, 1-11 (2017).

25. N. Lilienfein, C. Hofer, S. Holzberger, C. Matzer, P. Zimmermann, M. Trubetskov, V. Pervak, and I. Pupeza, "Enhancement cavities for few-cycle pulses," Opt. Lett. 42, 271-274 (2017).

26. M. Högner, V. Tosa, and I. Pupeza, "Generation of isolated attosecond pulses with enhancement cavities—a theoretical study,” New J. Phys. 19, 033040 (2017).

27. M. Högner, T. Saule, N. Lilienfein, V. Pervak, and I. Pupeza, "Tailoring the transverse mode of a high-finesse optical resonator with stepped mirrors," J. Opt. 20, 024003 (2018).

28. C. M. Heyl, S. N. Bengtsson, S. Carlström, J. Mauritsson, C. L. Arnold, and A. L'Huillier, "Corrigendum: Noncollinear optical gating (2014 New J. Phys. 16 052001)," New J. Phys. 16, 109501 (2014).

29. M. Louisy, C. L. Arnold, M. Miranda, E. W. Larsen, S. N. Bengtsson, D. Kroon, M. Kotur, D. Guénot, L. Rading, P. Rudawski, F. Brizuela, F. Campi, B. Kim, A. Jarnac, A. Houard, J. Mauritsson, P. Johnsson, A. L'Huillier, and C. M. Heyl, "Gating attosecond pulses in a noncollinear geometry," Optica 2, 563-566 (2015).

30. H. Vincenti and F. Quéré, "Attosecond Lighthouses: How To Use Spatiotemporally Coupled Light Fields To Generate Isolated Attosecond Pulses," Phys. Rev. Lett. 108, 113904 (2012).

31. K. T. Kim, C. Zhang, T. Ruchon, J.-F. Hergott, T. Auguste, D. M. Villeneuve, P. B. Corkum, and F. Quéré, "Photonic streaking of attosecond pulse trains," Nat. Photonics 7, 651-656 (2013).

32. T. J. Hammond, G. G. Brown, K. T. Kim, D. M. Villeneuve, and P. B. Corkum, "Attosecond pulses measured from the attosecond lighthouse," Nat. Photonics 10, 171-175 (2016).

33. T. Auguste, O. Gobert, T. Ruchon, and F. Quéré, "Attosecond lighthouses in gases: A theoretical and numerical 
Optics EXPRESS

study,” Phys. Rev. A 93, 033825 (2016).

34. E. Balogh, C. Zhang, T. Ruchon, J.-F. Hergott, F. Quere, P. Corkum, C. H. Nam, and K. T. Kim, "Dynamic wavefront rotation in the attosecond lighthouse," Optica 4, 48-53 (2017).

35. H. Carstens, S. Holzberger, J. Kaster, J. Weitenberg, V. Pervak, A. Apolonski, E. Fill, F. Krausz, and I. Pupeza, "Large-mode enhancement cavities," Opt. Express 21, 11606-11617 (2013).

36. S. Holzberger, N. Lilienfein, M. Trubetskov, H. Carstens, F. Lücking, V. Pervak, F. Krausz, and I. Pupeza, "Enhancement cavities for zero-offset-frequency pulse trains," Opt. Lett. 40, 2165-2168 (2015).

37. X. Gu, S. Akturk, and R. Trebino, "Spatial chirp in ultrafast optics," Opt. Commun. 242, 599-604 (2004).

38. L. von der Wense, B. Seiferle, M. Laatiaoui, J. B. Neumayr, H.-J. Maier, H.-F. Wirth, C. Mokry, J. Runke, K. Eberhardt, C. E. Düllmann, N. G. Trautmann, and P. G. Thirolf, "Direct detection of the 229th nuclear clock transition," Nature 533, 47-51 (2016).

39. C. Gaida, M. Gebhardt, T. Heuermann, F. Stutzki, C. Jauregui, and J. Limpert, "Ultrafast thulium fiber laser system emitting more than $1 \mathrm{~kW}$ of average power," Opt. Lett. 43, 5853-5856 (2018).

40. J. Zhang, K. Fai Mak, N. Nagl, M. Seidel, D. Bauer, D. Sutter, V. Pervak, F. Krausz, and O. Pronin, "Multi-mW, few-cycle mid-infrared continuum spanning from 500 to $2250 \mathrm{~cm}$-1, Light. Sci. Appl. 7, 17180 (2018).

41. A. E. Siegman, Lasers (University Science Books, 1986).

42. D. G. Voelz, Computational fourier optics - a MATLAB® tutorial (SPIE Press, Bellingham, Wash, 2011).

43. G. Scoles, Atomic and Molecular Beam Methods (Oxford University Press, 1988).

44. J. Weitenberg, P. Rußbüldt, I. Pupeza, T. Udem, H.-D. Hoffmann, and R. Poprawe, "Geometrical on-axis access to high-finesse resonators by quasi-imaging: a theoretical description,” J. Opt. 17, 025609 (2015).

45. I. Pupeza, E. E. Fill, and F. Krausz, "Low-loss VIS/IR-XUV beam splitter for high-power applications," Opt. Express 19, 12108-12118 (2011). 